\title{
Tangence
}

\section{Revenants ou la vie dans les livres}

\section{Daniel Canty}

Numéro 43, mars 1994

Babel et après : Paul Auster

URI : https://id.erudit.org/iderudit/025800ar

DOI : https://doi.org/10.7202/025800ar

Aller au sommaire du numéro

Éditeur(s)

Tangence

ISSN

0226-9554 (imprimé)

1710-0305 (numérique)

Découvrir la revue

Citer cet article

Canty, D. (1994). Revenants ou la vie dans les livres. Tangence, (43), 33-50. https://doi.org/10.7202/025800ar d'utilisation que vous pouvez consulter en ligne.

https://apropos.erudit.org/fr/usagers/politique-dutilisation/ 


\section{Revenants ou la vie dans les livres Daniel Canty}

Pendant un moment, j'ai caressé l'idée de mettre une épigraphe au début de Cité de verre. Une phrase de Wittgenstein: "Et cela a un sens aussi de parler de vivre dans les pages d'un livre".

\section{Paul Auster, L'art de la faim}

Whether in my book or not, every man is tabernacled in every other and he in exchange and so on in an endless complexity of being and witness to the uttermost edge of the world.

Cormac McCarthy, Blood Meridian

Paul Auster, dans une entrevue accordée en 1991 à JeanFrançois Chassay et Michel Biron de la revue Spirale, affirmait son goût pour la littérature du $\mathrm{XIX}^{\mathrm{e}}$ siècle $^{1}$, qui se manifeste dans ses ouvres par un traitement intertextuel massif du corpus, surtout américain mais aussi étranger, de cette période ${ }^{2}$. Je m'attarderai

1 Jean-François Chassay et Michel Biron, "Entre le western et Kafka", Spirale, décembre-janvier 1991, n 102 , p. 12 et 23: "Pour moi, le sommet de la littérature américaine se trouve au $x_{x}{ }^{e}$ siècle. Thoreau, Melville et Hawthorne sont essentiels, mais aussi Dickinson, Poe, Emerson. Ce sont de très grandes cuvres qui posent des questions très actuelles et qui ont inventé la littérature américaine".

2 Jusqu'à présent, les commentateurs de La trilogie new-yorkaise, au reste peu nombreux, ne se sont pas attardés à cette composante importante de l'œuvre d'Auster, et ont plutôt concentré leur attention sur la "déconstruction" du genre policier qui s'y effectuerait (voir Alison Russell, "Deconstructing The New York Trilogy: Paul Auster's Anti-Detective Fiction, Critique, hiver 1990, vol. XXXII, $\mathrm{n}^{\mathrm{O}} 2$, p. 71-85; et Norma Rowen, "The Detective in Search of the Lost Tongue of Adam: Paul Auster's City of Glass", Critique, éte 1991, vol. XXXII, $\mathrm{n}^{\circ} 4$, p. 224-233). L'article récent de William Lavender, "The Novel of Crtical Engagement: Paul Auster's City of Glass" (Contemporary Literature, 
plus particulièrement à un texte qui me semble exemplaire à cet égard: Revenants ${ }^{3}$, le deuxième volet de La trilogie new-yorkaise, à propos de laquelle Marc Chénetier affirmait qu'on pourrait "sans crainte [découvrir] [...] le grand livre de la littérature américaine dont, avec Whitman et Thoreau [...], Hawthorne, Melville et Poe écrivirent les chapitres les plus significatifs" " ${ }^{4}$ Non seulement la trace de ces auteurs se donne-t-elle à lire en palimpseste dans Revenants, mais encore permet-elle de considérer le roman en entier comme une glose sur les idéaux des transcendantalistes, rassemblés autour d'Emerson à Boston au milieu du XIX ${ }^{\mathrm{e}}$ siècle.

Lorsque Bleu, aux trois-quarts du roman, pénètre enfin dans la chambre de Noir, qui, dans le rôle de Blanc, l'a engagé pour se faire surveiller, sa déception est grande. Là où il espérait trouver une "image [...] [dont] il pensait qu'elle lui fournirait un indice précieux", il voit "qu'il n'y a rien" (p. 72), sinon des milliers de pages "vierges ou noircies, remplies à la machine ou à la main" et une poignée de livres dans une bibliothèque: Walden de Henry David Thoreau, Leaves of Grass de Walt Whitman, Twicetold Tales de Nathaniel Hawthorne, où fut pour la première fois en 1837 recueillie la nouvelle "Wakefield", "et quelques autres" (p. 73) volumes dont les titres ne sont pas précisés ${ }^{5}$. La bibliothè-

été 1993, vol. XXXIV, $\mathrm{n}^{\circ} 2$, p. 219-239), s'il fait montre de plus d'originalité, ignore pour la plus grande part cette dimension intertextuelle fondamentale de l'ouvre d'Auster. Seul Marc Chénetier, dans la "lecture" qui accompagne l'édition de poche de La trilogie new-yorkaise, en glisse un mot, en se contentant toutefois de quelques remarques de caractère programmatique (voir Marc Chénetier, "Le monde pseudonyme de Paul Auster", dans Paul Auster, La trilogie new-yorkaise, Arles, Actes Sud, Babel, 1991 (1987 et 1988), p. 431445).

3 Paul Auster, Revenants, Arles, Actes Sud, 1988 (1986), 87 p. Toute référence au texte sera désormais indiquée par le numéro de page entre parenthèses.

4 Marc Chénetier, op. cit., p. 440.

5 Il y a déjà, dans la description de la chambre de Noir, une référence intertextuelle à Poe. Bleu n'y trouve qu'un amas d'écritures et pense "On ne peut pourtant pas appeler ça une vie [...]. En fait, ça n'a pas de nom. C'est un nulle part, le territoire qu'on trouve au bout du monde" (p. 73). Arthur Gordon Pym, dans son voyage vers le pôle, aboutit au dernier territoire du monde. L'exploration de ses cavernes révélera que leur topographie, à l'image des parcours de Peter Stillman (père) à travers New York dans Cité de verre (Trilogie, op. cit., chap. 8) dessine la semblance des caractères d'un alphabet. Quinn ira même jusqu'à comparer explicitement sa découverte d'un motif dans les déambulations de Stillman aux indentations que découvrent Pym et son compagnon d'infortune, le mulâtre Peters, au fond d'un cul-de-sac au plus creux de ces cavernes du bout du monde (voir Edgar 
que de Noir, plutôt modeste, présente l'avantage de pouvoir être facilement reconstituée en dehors du livre. Le lecteur attentif, se gardant bien de commettre les mêmes erreurs que Bleu, s'y plongera pour trouver les images et les indices qui manquent au mur de Noir. Le narrateur a déjà mis le lecteur en garde quant au fait que Bleu pêche par inattention, lui qui préfère oublier le conseil de Thoreau, "Les livres doivent être lus avec autant de considération et de réserve qu'on a mis à les écrire" (p. 44), et abandonner sa lecture de Walden:

Ce qu'il [Bleu] ne sait pas, c'est que s'il arrivait à avoir la patience de lire ce livre dans l'esprit que sa lecture exige, sa vie entière commencerait à changer et petit à petit il parviendrait à une compréhension pleine et entière de sa situation - c'est-àdire de Noir, de Blanc, de l'affaire, de tout ce qui le concerne. Mais les chances perdues font autant partie de la vie que les chances saisies, et une histoire ne peut s'attarder sur ce qui aurait pu avoir lieu. (p. 45)

Le lecteur serait bien sot de ne pas appliquer le conseil de Thoreau à sa lecture de Revenants. Qu'il suive le texte comme Bleu suit Noir jusqu'à la librairie (p. 29-30), et aille se procurer lui aussi une copie de tous ces livres dont ce récit, "longue harangue à propos de rien du tout. (p. 45), semble n'être rien d'autre que la lecture, et peut-être parviendra-t-il, au contraire de Bleu, à "une compréhension pleine et entière de sa situation" de lecteur. $\mathrm{Si}$ "une histoire ne peut s'attarder sur ce qui aurait pu avoir lieu",

Allan Poe, The Narrative of Artbur Gordon Pym of Nantucket, New York, Penguin, 1986 (1838), chap. 23), pour ensuite rejeter cette analogie en se cautionnant de l'impermanence du message tracé par Stillman dans son mouvement. Toujours est-il que c'est la même équivoque qui persiste devant un message comme l'autre, à savoir qu'il est impossible de déterminer si la production de ces signes est le résultat du travail intentionnel d'un auteur ou seulement l'illusion de leur lecteur, dont l'interprétation amène une distorsion dans la perception de faits qui figurent dans leur insignifiance le principe apparent de production des phénomènes les plus communs de la nature, c'est-à-dire le hasard. Les incertitudes de Quinn rejoignent celles de Bleu à travers la médiation de l'œuvre de Poe. Bleu reste à l'image de Pym et de Quinn interdit devant une accumulation de signes qui défie, bien que d'une manière toute différente que pour ses contreparties, le sens. Si, dans un cas comme dans les autres, c'est de l'énigme de leur production que les signes tiennent leur mystère, pour Pym comme Quinn, c'est leur provenance qui questionne le bon sens, alors que pour Bleu c'est la raison même de leur production. Cependant, dans chacun des cas, c'est toujours un manque, qu'il soit de raison ou d'intention, qui menace d'engouffrer le sens dans son vide. 
il est possible que le lecteur, lui, dispose de tout son temps. Il se dira, comme Bleu, que "S'il ne peut lire ce que Noir écrit, il pourra au moins lire ce qu'il lit" (p. 30).

Peut-être cela compensera-t-il pour l'inaccessibilité du livre imaginaire dont les feuillets s'empilent dans la chambre de Noir? Qui sait, peut-être ce livre est-il le "grand livre de la littérature américaine" dont parle Chénetier? Nous ne disposons que de la description des feuillets de ce livre et nous devrons nous débrouiller avec cela. Un livre n'étant jamais fait que d'autres livres, il nous sera peut-être possible de reconstruire la trame de ce manuscrit secret grâce aux ouvrages de la bibliothèque de Noir. Pour ce faire, il faudra nous aider non seulement des trois ouvrages dont le titre nous est révélé, mais encore des indices, éparpillés dans le texte, qui nous permettent de deviner quels autres livres on pourrait trouver sur les tablettes de Noir.

Tout semble indiquer que l'erreur fondamentale de Bleu consiste à postuler que le monde des textes et celui des faits sont incommensurables, ce qui le porte à conclure qu'“Il n'est pas possible qu'un homme comme Noir existe" (p. 54) puisque celui-ci "ne fait rien, [...] reste simplement assis dans sa chambre à écrire" (p. 53). Pourtant, la fiction et le réel, dans le monde de Bleu, s'entre-pénètrent, tant et si bien qu'il devient difficile, sinon impossible, de les distinguer. L'épisode où Bleu, à l'été 1948, trouve "enfin le courage d'agir" et décide de se déguiser en Jimmy Rose, "vieillard qui avait coutume de mendier dans son quartier lorsqu'il était jeune garçon" (p. 55), est à ce titre révélateur. Jimmy Rose, qui fait partie de l'histoire personnelle de Bleu, fait aussi partie du monde de la fiction, puisque que c'est là le titre d'une nouvelle de Herman Melville, qui raconte l'histoire d'un richissime et expansif gentilhomme du même nom tombé en disgrâce. D'abord, Rose décide de se retirer du monde en se réfugiant dans une vieille maison qui évoque étrangement celle de Fanshawe dans $L a$ chambre dérobée ${ }^{6}$. Mais d'un naturel "trop foncièrement bien-

6 D'ailleurs, il semblerait qu'Auster reprenne les éléments, dans le troisième volet de la Trilogie, de l'épisode de la nouvelle de Melville où le narrateur tente d'aller rendre visite au reclus. Comme Fanshawe, Rose refuse de s'identifier, et, qui plus est, sa voix et son comportement sont méconnaissables. Devant l'insistance du narrateur à vouloir pénétrer dans sa demeure, Rose se voit obligé de le menacer, exactement comme Fanshawe, d'un revolver glissé par le trou de serrure de sa porte: 
veillant et bénin pour qu'une cause quelconque pût faire de lui un misanthrope " 7 , Rose décide finalement de revenir, sous les allures d'un mendiant, portant redingote et pantalons étriqués, au monde qu'il avait connu étant riche. Il continue de fréquenter les salons où il en profite pour faire provision de biscottes et de toasts. Il ne perd cependant ni sa politesse ni sa galanterie. Le Jimmy Rose de Bleu, comme celui de Melville, "n'est pas tant un miséreux scrofuleux qu'un sage illuminé, un saint apôtre de la pénurie vivant dans les marges de la société „ (p. 55).

Malgré les changements que subit son apparence extérieure, Jimmy Rose reste fidèle à lui-même. Les préoccupations morales de Melville semblent rejoindre celles des transcendantalistes, pour qui l'âme, siège de l'identité et fondement de la dignité humaine, seule restait inchangée au milieu des métamorphoses du corps et du monde. Cependant, celle-ci n'était pas donnée une fois pour toutes; aussi fallait-il, par ses actions, la mettre au monde. Il fallait que chaque geste provienne d'une nécessité intérieure, garante de son authenticité ${ }^{8}$. Dès lors, l'action devenait un moyen de connaissance, par lequel l'homme se rapprochait, en l'extériorisant, de sa nature véritable. La question de l'authenticité rejoint chez les transcendantalistes des préoccupations sur la nature fondamentalement religieuse et sacrée du réel. Pour eux, chaque homme qui vivait selon son âme vivait de manière religieuse et profondément morale. Jimmy Rose réalise l'idéal transcendantaliste en continuant à donner alors même qu'il ne possède plus rien:

- Quittez les lieux, sinon...

Sur quoi j'entendis ferrailler contre l'énorme serrure; mais non pas un bruit de clé: quelque chose comme l'introduction d'un petit tube creux dans le trou de la serrure. Horrifié, je m'enfuis de toute la vitesse de mes jambes.

(Herman Melville, Moi et ma cheminée, Paris, Seuil, coll. "Points", 1984, p. 117) On verra que c'est une pratique commune pour Auster que de reprendre certains textes pour en explorer les virtualités, c'est-à-dire de retourner aux fourches où le récit menaçait de bifurquer pour emprunter les branches de cet "arbre des récits" que figure l'intertexte. Ainsi en va-t-il avec le récit de Melville: dans le roman d'Auster, le narrateur ne se sauve pas mais reste. Par le fait même, Auster encourage l'exploration par le lecteur des possibilités non réalisées de ses propres récits.

7 Melville, op. cit., p. 119.

8 Pour ce qui est de la philosophie transcendantaliste, je me référerai, tout au long de ce texte, à l'ouvrage de Catherine Albanese, Corresponding Motion: Transcendental Religion and the New America, Philadelphia, Temple University Press, 1977, 210 p. 
Encore que, dans ton indigence, tu n'eusses pas un penny à donner aux pauvres, Jimmy, tu faisais, toi, l'aumône aux riches. Car le mendiant qui marmonne au coin de la rue attend son pain moins avidement que le cour vaniteux son compliment. L'avidité des riches dans la satisfaction et l'avidité des pauvres dans le besoin, c'est tout un. Et c'est cela, je pense, que devait se dire Jimmy Rose. ${ }^{9}$

Le Jimmy Rose de Bleu, comme on pouvait s'y attendre, est tout aussi généreux. Ainsi, au moment de sa première rencontre avec Noir, il reçoit de lui un peu de monnaie et ne manque pas de dire à son bienfaiteur, "Que Dieu vous bénisse" (p. 56). Ce dernier est touché par le sentiment et l'en remercie. Bleu s'empresse d'ajouter, en bon transcendantaliste, "Dieu nous bénit tous" (p. 56) ${ }^{10}$.

La question de l'authenticité n'est pas seulement centrale dans la pensée transcendantaliste. Elle travaille aussi les textes de Thoreau et de Whitman. La deuxième rencontre de Jimmy Rose/Bleu avec Noir nous renvoie d'emblée à la bibliothèque de Noir. En effet, celui-ci aborde Rose et lui demande: "Est-ce qu'on vous a jamais dit que vous ressemblez parfaitement à Walt Whitman?» (p. 56). Le projet de Whitman, en écrivant Leaves of Grass, est de beaucoup semblable à celui des transcendantalistes, qui croyaient comme lui que l'idéal démocratique de la nation américaine ne pourrait trouver à se réaliser pleinement qu'à travers le développement des individus. Comme l'écrit Whitman: "While the ambitious thought of my song is to help the forming of a great aggregate Nation, it is, perhaps, altogether through the forming of myriads of fully develop'd and enclosed individuals" "11.

Il s'agissait, pour l'auteur de Leaves of Grass, de confondre sa vie avec la composition de son livre, qu'il étendra et modifiera jusqu'au jour de sa mort. Whitman voulait construire un poème

9 Melville, op. cit., p. 125.

10 Paradoxalement, on pourrait affirmer que le geste de Bleu/Rose tire son authenticité de la vraisemblance de la performance de Bleu. Il s'agirait donc moins ici d'incarner son âme que celle d'un autre dont on choisit de revêtir le masque. Les conséquences heuristiques d'un tel acte me semblent précisément correspondre à celles des situations explorées dans Revenants.

11 Walt Whitman, Leaves of Grass, New York, Bantam, 1983, p. 453. Traduction personnelle: "Alors que l'ambitieuse visée de mon chant est de contribuer à la formation d'une grande Nation collective, c'est peut-être, somme toute, à travers la formation de multitudes d'individus entièrement développés et autonomes". 
qui partagerait avec sa personne une forme logique, et dont la croissance au fil des ans doublerait la sienne. Dès lors, la poésie n'est plus tellement affaire de métaphore que d'homologie, de "correspondance fonctionnelle", pour parler dans des termes chers à Joseph McElroy ${ }^{12}$, et, à la fin, cela n'a peut-être plus grand-chose à voir avec la littérature ${ }^{13}$. Whitman écrivait :

"Leaves of Grass" indeed (I cannot too often reiterate) has mainly been the outcropping of my own emotional and other personal nature - an attempt, from first to last, to put a Person, a human being (myself, in the latter half of the Nineteenth Century, in America), freely, fully and truly on record. [...] But it is not on "Leaves of Grass" distinctively as literature, or a specimen thereof, that I feel to dwell, or advance claims. No one will get at my verses who insists upon viewing them as a literary performance, or attempt at such performance, or as aiming mainly toward art or aestheticism. ${ }^{14}$

12 Voir Joseph McElroy, "Fiction - domaine de croissance", La main de singe, $\mathrm{n}^{\circ} 7$, p. 21-27, 1993, et aussi "Neural Neighborhoods and Other Concrete Abstracts", Tri-Quarterly, $\mathrm{n}^{\circ} 34,1975$, p. 201-217.

13 "À la fin, ça n'a peut-être pas grand-chose à voir avec la littérature", Paul Auster, "Conversation avec Joseph Malla " L'art de la faim, Arles, Actes Sud, 1992 (1982), p. 254.

14 Whitman, op.cit., p.456. Traduction personnelle:

"Leaves of Grass" en fait (je ne peux assez souvent le répéter) a surtout été l'affleurement de ma propre nature émotionnelle et personnelle - une tentative, du début à la fin, de mettre une Personne, un être humain (moi-même, dans la seconde moitié du dix-neuvième siècle, en Amérique), librement, complètement et vraiment au registre. [...] Mais ce n'est pas sur "Leaves of Grass" distinctement comme littérature, ou comme un de ses spécimens, que je veux me pencher, ou dont je me réclame. Nul ne saisira mes vers s'il insiste pour y voir une performance littéraire, ou une tentative d'une telle performance, ou comme visant principalement à l'art ou l'esthétisme.

Il faudrait dresser un parallèle entre la tentative de Whitman et ce que les "cyberpunks" appellent le "flatlining ( $c f$. William Gibson, Neuromancer, New York, Ace, 1984), c'est-à-dire la substitution du support traditionnel de la subjectivité, le corps, par un dispositif de stockage d'informations où, après sa mort, le sujet perdure sous forme d'influx électriques. Les cyberpunks, il va sans dire, s'inspirent de la croyance - usitée dans certaines branches des sciences cognitives - en la traductibilité de toutes choses en termes d'informations. Le projet de Whitman, qui vise à s'enregistrer, grâce au support de la page, șous forme de poème, préfigurerait ainsi certaines des questions que se posent aujourd'hui les spécialistes de l'intelligence artificielle. On verra donc dans Leaves of Grass une forme archaïque de "flatlining", le terme ne se référant plus tellement ici à la ligne plate qui, sur les cardiographes, marque l'horizon de la vie, qu'à l'horizontalité indépassable des lignes du poème. 
40

Paradoxalement, l'investissement narcissique du sujet dans son texte semble mettre au jour "le réseau de collaboration qu'est l'expérience humaine " ${ }^{15}$. En effet, le livre de Whitman appelle la collaboration du lecteur, qu'il puisse redevenir "domaine de croissance", le savoir du lecteur s'engageant dans un processus de développement semblable à celui de Whitman. Ce processus réactive, et ce à travers le point de vue de chacun, la subjectivité de son auteur à même l'objet inanimé qu'est le poème et poursuit son projet par-delà l'horizon de sa mort. L'ambition de Whitman " to formulate a poem whose every thought or fact should directly or indirectly be or connive at an implicit belief in the wisdom, health, mystery, beauty of every process, every concrete object, every human or other existence, not only consider'd from the point of view of all, but of each " 16 trouve sa realisation à travers la croissance de la constellation d'individualités que représente l'ensemble des lecteurs du poème. En confondant sa personne à ses poèmes, Whitman a fait de la lecture le principe et la source de sa vie.

Le lecteur du texte d'Auster s'engage dans un processus de croissance similaire à celui qu'est supposé impliquer la lecture de Leaves of Grass. L'abondance des références intertextuelles dans Revenants aboutit à une croissance exponentielle du texte, dont la compréhension nécessite le recours du lecteur à toutes les ressources de son encyclopédie ${ }^{17}$, et sans aucun doute à une extension de celle-ci. Le lecteur qui présuppose que ces références font système entre de plain-pied dans le domaine de la métaphysique, de l'invérifiable: il ne peut qu'inventer des histoires, des théories du texte dont il suppose qu'elles rendent compte avec justice de leur objet. Dès lors, il postule la manifestation dans le texte d'une intentionnalité, d'une visée, consciente ou non, et par laquelle s'inscrit en négatif la présence d'un sujet. À ce titre, il

15 Joseph McElroy, cité et traduit par Pascale Guilpain, "Joseph McElroy, poète du neuronal ", Europe, "Écrivains des États-Unis", mai 1990, n 733 , p. 59.

16 Whitman, op. cit., p. 455. Traduction personnelle : l'ambition de Whitman "de formuler un poème dont chaque pensée ou fait devrait directement ou indirectement être ou suggérer une croyance implicite dans la sagesse, la salubrité, le mystère, la beauté de chaque processus, chaque objet concret, chaque existence humaine ou autre, non seulement considérée du point de vue de tous, mais aussi de chacun".

17 Voir Umberto Eco, Lector in fabula, Paris, Grasset, Le livre de poche, 1985 (1979), $315 \mathrm{p}$. 
ressemble à Bleu, qui ne cesse de croire que la situation dans laquelle il se trouve a été pensée par quelqu'un d'autre, comme en témoignent les références répétées à l'existence possible d'un "système" (p. 30), d'un "maître au-dessus de lui" (p. 37) (Bleu se voit comme "une poupée de chiffon qui a perdu sa bourre" (p. 37), d'une "ombre [qui] continue à planer sur lui " (p. 43), etc.

Mais revenons pour l'instant au projet de Whitman, qui repose sur la croyance en une équivalence possible entre la forme du poème et celle du sujet, et qui permet ainsi d'aborder la doctrine de la correspondance, centrale à la pensée des transcendantalistes, et selon laquelle "chaque partie du monde était un miroir pour chaque autre partie du monde " ${ }^{18}$. Chacune des diverses facettes du projet transcendantaliste répond à la nécessité de mettre au jour la correspondance existant entre toutes les parties de la création, ce qui sert pour témoigner d'une volonté directrice sous les aspects du monde, elle-même garante de sa sacralité. Il suffira de considérer le rapport qu'entretiennent chez Whitman ou chez les transcendantalistes la quête de l'authenticité personnelle et la recherche d'une identité nationale américaine, pour voir comment le local s'intriquait au global, le microcosme au macrocosme ou encore l'intériorité à l'extériorité dans l'idéal transcendantaliste.

Comme l'a montré Catherine Albanese dans son ouvrage Corresponding Motion, c'est surtout à travers des actes de langage, en essayant de trouver un mode d'expression qui traduirait à la fois la réalité changeante qui les entoure et leur expérience intime de cette réalité, que les transcendantlistes ont travaillé à exposer la correspondance. C'est donc dire que le langage était pour les transcendantalistes le mode privilégié de l'action. L'importance qui lui était accordé tant par les transcendantalistes que par Whitman, qui révoque une vie "normale " pour s'investir dans la poésie, repose sans aucun doute sur son statut ambigu, celui-ci se trouvant aà la fois à l'intérieur et à l'extérieur du cerveau "19. Il se pourrait bien que l'" infirmité de notre nature " évoquée dans une citation de Walden ${ }^{20}$ reprise dans Revenants fasse référence à notre statut d'êtres de langage, qui fait que nous vivons à la fois

18 Albanese, op. cit., p. 7.

19 Guilpain, op. cit., p. 62.

20 Henry David Thoreau, Walden and Civil Disobedience, New York, Harper and Row, 1965, $271 \mathrm{p}$. 
42

dans le monde et dans notre tête, où nous nous racontons sans cesse l'histoire de notre propre vie ${ }^{21}$ :

Nous ne sommes pas où nous sommes [...] mais dans une fausse position. Par une infirmité de notre nature nous supposons une affaire dans laquelle nous nous mettons; du coup nous sommes dans deux affaires à la fois et il est doublement difficile d'en sortir. (p. 52)

La collaboration de l'auteur et du lecteur se faisant, dans le cas de Whitman, à travers le poème, on dira qu'elle est rendue possible par le dédoublement qu'implique notre usage du langage. Tout se passe comme si, en lisant Leaves of Grass, qui se présente comme une extériorisation de la conscience de Whitman, le lecteur se plaçait à même son cerveau et devait reprendre le fil de l'histoire de Whitman là où il l'a laissé, lui seul pouvant activer les réseaux de sens et de sensation stockés dans les poèmes, refaire les branchements déconnectés de ses synapses. Si l'on peut, comme j'ai tenté de le montrer, identifier l'acte de lecture à la continuation du projet de Whitman, et donc à celle de sa vie, qui se confond à ce projet, on dira alors que le lecteur tend à réaliser une sorte de correspondance proche de celle qui a été rêvée par les transcendantalistes. Cependant, la croissance du savoir du lecteur ne peut se réaliser, de peur de révoquer sa singularité inaltérable, en parfaite synchronie avec celle d'un autre: elle peut tout au plus en suivre les rythmes, recomposer des patrons qui lui permettront d'approcher de ce que l'autre pouvait être sans jamais l'atteindre. La correspondance est donc nécessairement un processus inachevé.

L'intérêt d'une telle conception pour notre lecture de Revenants trouve à s'illustrer dans la conversation que Noir tient à Bleu concernant le cerveau de Walt Whitman. L'intertexte est en quelque sorte une forme littéraire de la correspondance et, conséquemment, c'est du croisement de plusieurs récits que Noir tirera le sien. D'ailleurs, l'endroit où se tiennent Rose et Noir est déjà une espèce de noeud interdiscursif : non seulement c'est la rue où Whitman a imprimé Leaves of Grass mais encore Thoreau, la veille de sa visite à la demeure de Whitman sur Myrtle Street, s'y

21 "Tout individu complet [...], toute personne possédant une identité cohérente, est constamment en train de se raconter l'histoire de sa vie d'un instant à l'autre - en suivant le fil de sa propre histoire", Paul Auster, paraphrasant Oliver Saks, L'art de la faim, op. cit., p. 289. 
est-il rendu pour écouter le sermon de Henry Ward Beecher, abolitionniste qui partageait avec Thoreau, Whitman et les transcendantalistes leur croyance en la dignité fondamentale de l'hom$\mathrm{me}^{22}$. Le premier des récits de Noir concerne la croyance de Whitman en la phrénologie, discipline para-scientifique qui postulait la correspondance entre la forme extérieure du cerveau et le caractère de celui à qui ce cerveau appartient. L'organe de Whitman aurait après sa mort été légué à un laboratoire où on l'aurait échappé sur le sol, ce qui eut pour conséquence de le faire voler en éclats. Le deuxième raconte la visite de Thoreau et Alcott (un des transcendantalistes) à la demeure de Whitman. Noir établit une correspondance entre le cerveau éclaté de Whitman et la merde remplissant un seau traînant sur le sol de sa chambre, après quoi il conclut que cela ne sert pas à grand chose "d'essayer de se mettre dans la peau d'un auteur":

si on veut bien y réfléchir, il y là une certaine ressemblance de forme. Je veux parler des bosses et des circonvolutions. Il y a un lien indéniable. Le cerveau et les entrailles, l'intérieur de l'homme. On parle toujours d'essayer de se mettre dans la peau d'un auteur pour mieux comprendre son ouvre. Mais finalement il n'y a pas grand-chose à découvrir - du moins pas grand-chose qui differe de ce qu'on trouverait chez n'importe qui d'autre. (p. 60)

Pourtant, Noir «aime savoir comment vivent les écrivains, surtout ceux d'Amérique. Ça [...] [l']aide à comprendre des choses" (p. 60). Bleu, lui, est "un fervent de Vrai Détective et il s'efforce de ne jamais en manquer un mois" (p. 15). Entre les histoires qui se donnent pour vraies de Bleu et les histoires d'écrivains de Noir, il n'y a pas tellement de différence. Qui plus est, il n'y a pas tellement de différence entre toutes ces histoires et celle que racontent les écrivains. L'usage qui est fait, tout au long de Revenants, de la nouvelle de Nathaniel Hawthorne, "Wakefield" 23 , le dernier des récits racontés par Noir à Rose, montre que la

22 La référence à Abraham Lincoln (p. 59) me semble renvoyer elle aussì à ce thème, de même que l'épisode où Bleu va au stade et voit jouer le premier joueur noir des ligues majeures, Jackie Robinson (p. 39-40), dont la performance représente une sorte de manifestation en faveur de la race noire, capable de se mesurer et de dépasser le blanc à son propre jeu, ce par quoi s'affiche sa dignité.

23 Nathaniel Hawthorne, "Wakefield", Selected Tales and Sketches, New York, Penguin, 1987, p. 149-158. 
barrière entre les "faits" et les "histoires", pour employer des termes qui reviennent tout au long du roman, n'est pas si étanche qu'on pourrait le penser. La manière dont "Wakefield" contamine la vie de Bleu aurait dû le pousser à réviser son jugement sur le statut ontologique de la fiction.

Lorsque l'affaire commence, Bleu donne un coup de fil à sa future femme pour lui annoncer qu'il s'en va "under cover" 24 pour "un petit moment" (p. 9). Wakefield, au moment où il quitte sa femme, prétexte un court voyage d'affaires à la campagne, dont il ne précise pas plus la durée. En fait, il va s'isoler, sans raison, dans la rue d'à côté, où il retardera pendant vingt ans le moment de son retour, de la même manière que Bleu, décidant d'abord de ne pas appeler sa femme (p. 11), attendra si longtemps qu'il ne lui sera plus possible de la rejoindre. Le processus de transformation dans lequel s'engage Bleu ("Je change, se dit-il. Petit à petit, je ne suis plus le même"(p. 21)) bifurque de celui de Wakefield au moment où il croise sa femme dans la rue: dans "Wakefield", celle-ci, restée "veuve", ne reconnaît pas son mari, alors que Bleu méritera une bonne râclée de la part de sa femme et ne pourra plus jamais revenir à ce qu'il était. Là où la nouvelle de Hawthorne acceptait la réversibilité des phénomènes, l'histoire de Bleu aligne des événements dont l'irréversibilité s'affiche sans équivoque dès l'épisode de la rencontre avec celle qu'il sera désormais impossible de continuer à appeler la future $\mathrm{M}^{\mathrm{me}}$ Bleu (p. 45-47).

Les parallèles entre les deux textes ne s'arrêtent pas là: j'ai déjà évoqué les présomptions métaphysiques de Bleu, qui se sent manipulé du dehors et du dessus, intégré à un système qui le dépasse. Or, on peut lire dans "Wakefield": "an influence, beyond our control, lays its strong hand on every deed which we do, and weaves its consequences into an iron tissue of necessity "25. Par ailleurs, le narrateur de "Wakefield" présente la nouvelle comme

24 C'est ce qu'on peut lire dans l'édition anglaise (Paul Auster, Gbosts, New York, Penguin, Contemporary American Fiction, 1986, p. 9). Comme Marc Chénetier (op. cit) le faisait remarquer, on pourrait lire cette phrase comme signifiant que Bleu part se mettre sous le couvert d'un livre.

25 Hawthorne, op. cit., p. 155. Dans la traduction de Marc Logé (dans La vieille fille blanche et autres contres fantastiques, Paris, Stock, 1926, p. 25-37), on peut lire: "Une influence qui dépasse notre contrôle pose sa main vigoureuse sur chacun de nos actes et tisse ses conséquences en un tissu de fer. (p. 33). 
étant tirée d'un fait divers et le cas Gris, résolu par Brun et Bleu alors qu'ils travaillaient ensemble (p. 13-14), a toutes les caractéristiques d'un fait divers et constitue une variante de plus de "Wakefield". Ici, c'est l'amnésie de Gris, maintenant appelé Vert, qui lui permet de remarier sa femme, comme celle de Wakefield, une fois celui-ci revenu à la maison, redevient épouse, de veuve qu'elle était. Revenants dresse la carte des points où la fiction rejoint le réel et où celui-ci bifurque de celle-là, tant et si bien qu'il devient difficile de distinguer la vie qui se fait dans les livres et le langage de celle qui se passe dans le monde.

Le narrateur de "Wakefield" ressemble aussi à celui de Revenants. L'un comme l'autre parlent au "nous" et se présentent comme une sorte de consience désincarnée qui suivrait le personnage principal. On peut lire, par exemple, chez Hawthorne: "We must hurry after him [Wakefield], along the street, ere he lose his individuality " ${ }^{26}$. Chez Auster, ce sont les références constantes à "ce que nous savons" ("à partir de ce moment nous ne savons plus rien" (p. 87)) qui suggèrent une position narrative similaire. Toujours est-il que le narrateur d'Auster, comme celui de Hawthorne, qui a déjà lu le fait divers, semble détenir des informations inaccessibles à Bleu ("Certes, Bleu est loin de se douter que l'affaire durera des années" (p. 9)). Cela porte à considérer le récit comme la reprise par la pensée d'une expérience déjà achevée dont l'incipit décrirait l'espace substrat ${ }^{27}$, comme dit René Thom: "Le lieu: New York; le temps : le présent; aucun des deux ne changera jamais" (p. 7). Une fois ces conditions initiales ${ }^{28}$ posées, le narrateur ne fait rien de plus que dérouler une série d'énoncés observationnels qui décrivent le comportement du sujet expérimental "Bleu". Cela escompterait pour le caractère abstrait des nominations (des noms de couleur) et la désinvolture

26 Ibid., p. 151. Traduction: "Il nous faut presser le pas derrière lui le long de la rue, de crainte qu'il ne perde son individualité et se fonde dans la grande masse de la vie de Londres. (Logé, op. cit., p. 28).

27 "Les phénomènes qui sont l'objet d'une discipline [...] donnée apparaissent comme des accidents de formes définies dans un espace donné que l'on pourrait appeler l'espace substrat de la morphologie étudiée" (René Thom, Paraboles et catastrophes, Paris, Flammarion, Champs, 1983, p. 5.)

28 Il vaut la peine de citer la définition que l'on donne de ce terme en épistémologie: il y sert, selon Alan Chalmers, à désigner les "ensembles [...] [d']énoncés décrivant précisément la configuration à l'étude" (Qu'est-ce que la science?, Paris, La Découverte, Le livre de poche, biblio essais, 1987 (1982), p. 32). 
46

avec laquelle le narrateur souligne le caractère arbitraire des désignations et des localisations ("L'adresse n'a pas d'importance. Disons qu'il s'agit de Brooklyn Heights, par exemple" (p. 9)), ce qui rejoint en outre la stratégie narrative de Hawthorne: "What sort of man was Wakefield? We are free to shape out our own idea, and call it by his name "29. Le savoir du lecteur reprendra le processus de croissance qui a permis au narrateur d'arriver à conter son récit.

C'est non seulement au niveau de la narration, mais encore à celui des événements racontés, que Revenants se présente comme la relation d'une expérience. Les deux sens de ce mot, biographique et scientifique, trouvent au niveau de la diégèse à se confondre, les personnages sacrifiant toute espérance de mener une vie normale au profit d'une "situation artificielle" qui semble "construite en vue d'explorer et de tester [...] [une] théorie " ${ }^{30}$, comme le héros de Hawthorne s'engage dans ce que son auteur a appelé un "système" "31. On peut supposer que Noir, en engageant volontairement Bleu, déguisé en Blanc, pour se faire surveiller, obéit à une idée directrice, qu'il y a là un projet, orienté, consciemment ou non, par une visée qu'il nous s'agirait d'élucider. Lorsque Noir visite Bleu sous la gouverne de Blanc, il ne fait rien d'autre que lui décrire les conditions initiales de l'expérience, c'est-à-dire énumérer les énoncés sur lesquels se règlera son déroulement (voir p. 7-8). Noir cherche probablement à prouver quelque chose et pour cela il met sur pied un dispositif qui forcera Bleu à entrer dans le jeu de la confirmation. Cela ne s'arrête pas là, car le lecteur est à son tour appelé à trouver ce dont il s'agit, les tenants et les aboutissants de cette théorie secrète qui guide les agissements de Bleu et de Noir.

Comme l'affirmait Paul Auster, Revenants c'est "L'étang de Walden au cour de la ville" 32 et l'on comparera donc le projet de Noir avec celui de Thoreau. Cependant, il semblerait que Noir, plutôt que de se retirer du monde pour vivre dans les bois, s'isole dans les textes, entraînant Bleu à sa suite. Il faut souligner ici une

29 Hawthorne, op. cit., p. 150. Traduction: "Quel espèce d'homme était donc ce Wakefield? Nous sommes libres de former sur lui notre propre idée que nous affublerons ensuite de son nom" (Logé, op. cit., p. 26).

30 Chalmers, op. cit., p. 194.

31 Hawthorne, op. cit., p. 154.

32 L'art de la faim, op. cit., p. 252. 
parenté avec la tentative de Thoreau vis-à-vis des communautés expérimentales des Transcendantalistes, Brooks Farm et Fruitlands, et avec son projet de correspondance, par lequel la présence de l'autre fait retour au milieu de l'isolation la plus profonde. Auster disait justement qu' "on ne commence à comprendre sa relation aux autres que lorsqu'on se retrouve seul. 33 et on peut lire, dans Walden: "Not till we are lost, in other words, not till we have lost the world, do we begin to find ourselves, and realize where we are and the extent of our relations $" 34$.

Pour tenter d'élucider la théorie de Noir, il faut revenir à ce qu'il dit des écrivains à Rose. Après avoir parlé de son intérêt pour la vie des écrivains américains, il poursuit en affirmant que "L'écriture est une occupation solitaire qui accapare votre vie. Dans un certain sens, un écrivain n'a pas de vie propre. Même lorsqu'il est là, il n'est pas vraiment là" (p. 61). Si un écrivain "n'a pas de vie propre ", c'est qu'il n'existe que dans son rapport à la textualité, aux récits, et à la lecture que l'on peut faire de ces récits. Il y a là l'idée que c'est la présence de ses textes dans le regard de cet autre que constitue le lecteur qui donne vie et présence à l'écrivain, dont le lecteur imagine l'activité à l'origine du texte qu'il est en train de lire: la vie de l'écrivain ne se trouve pas tellement dans le monde que dans les livres. On ne peut pas se mettre dans la peau d'un auteur mais dans ses textes, qui tirent leur substance vitale d'autres textes. L'auteur est l'origine perdue du texte, dont les récits viennent recouvrir l'absence. Sous les couches des corps et des textes, il n'y a jamais que d'autres tissus, que d'autres textes. L'auteur se présente comme une béance qui rôde au milieu du texte, comme une sorte de "conscience en oignon". Comme l'explique Philippe Breton: "L'oignon n'a pas d'intériorité, il est tout entier fait d'extériorités superposées, enlevez lui sa peau externe, vous rencontrerez une autre peau et ainsi de suite". Auster affirmait: "Il est impossible de savoir ce que pense quelqu'un d'autre. Nous n'apercevons que les surfaces : les yeux,

33 Ibid., p. 289-290.

34 Walden, op. cit., p. 128. La traduction de Jeanne-Chantal et Thierry Fournier (Walden ou la vie dans les bois, Lausanne, L'Âge d'homme, 1985, p. 151) se lit comme suit: "Ce n'est qu'une fois que nous nous sommes perdus, autrement dit, lorsque nous avons perdu le monde, que nous commençons à nous trouver nous-mêmes, que nous réalisons là où nous sommes et l'étendue infinie de nos relations ". 
le visage, le corps" ${ }^{35}$, les peaux de l'oignon... Tout cela suggère que l'écrivain n'écrit pas tellement les histoires mais qu'il y réagit en les mêlant et les recomposant, qu'il est agi par celles-ci. Les textes comme les êtres "n'existent pas par eux-mêmes mais uniquement dans leurs rapports mutuels " 36 , dans l'échange sans fin de leur valeur et de leur identité. La fiction se monnaie pour du réel, le faux pour du vrai, comme l'a montré notre lecture croisée de la nouvelle de Hawthorne et du roman d'Auster.

Je résumerai le projet de Noir, qui peut subsumer la conception de l'écriture d'Auster, en disant qu'il cherche à éprouver les conditions minimales de sa propre réalité, c'est-à-dire à se placer dans le regard d'un autre, en l'occurrence Bleu, qui lui servira à se prouver à lui-même sa propre existence. Un constat existentiel se dégage à la lecture de Revenants qui permet de dire d'un texte qu'il n'existe que dans la mesure où il peut être lu, ou d'un homme qu'il n'est réel que dans la mesure où il peut être perçu. Il s'ensuit que ce qui existe est ce qui peut être connu... On peut se représenter le sujet (et, partant, l'écrivain) comme une sorte de béance qu'on se voit dans l'impossibilité de connaître autrement qu'en épluchant les surfaces qui l'entourent. Noir se présente justement comme "rien de plus qu'une sorte de manque, un trou dans la texture des choses, et une histoire peut tout aussi bien qu'une autre chose combler ce creux" (p. 20). C'est pourquoi il demande à Bleu de rédiger un rapport hebdomadaire qui s'en tient aux faits de surface qui constituent l'action de "prendre connaissance" de Noir. Cela posera d'emblée problème à Bleu, qui se verra forcé de recourir, pour combler le vide créé par l'impossibilité de connaître Noir, à toutes sortes d'histoires qu'il se racontera à lui-même, de la manière la plus involontaire du monde. Bleu découvre à ses dépens qu'"il n'y a pas de limite aux récits qu'il peut concocter» (p. 20). Symptomatiquement, ses rapports, suite d'énoncés observationnels qui invitent à toutes les dérives (voir les difficultés que Bleu éprouve à camper les faits de l'affaire, p. 22-23), devront être déposés dans une boîte postale dont le numéro, 1001, renvoie à cet enchaînemenent potentiellement

35 L'Art de la faim, op, cit., p. 288.

36 Philippe Breton, L'utopie de la communication, Paris, La Découverte, essais, 1992, p. 55. Précisons qu'il s'agit d'une métaphore employée par Alan Turing dans son article célèbre, "Les ordinateurs et l'intelligence" (dans Alan Ross Anderson (dir.), Pensée et macbine, Paris, Champ Vallon, 1983). 
infini de temps et de récits que noue Schehrazade pour son futur époux.

Revenants illustre qu'il est à strictement parler impossible de s'en tenir aux faits. Bleu tente d'adopter une stratégie d'induction et, conséquemment, se heurte aux problèmes épistémologiques qui y sont liés. Pour l'inductiviste naiff, la vérité d'une hypothèse se mesure à l'aune de l'observation. Il doit procéder sans préjugés à une accumulation d'observations qui forment la base sur laquelle reposera son savoir théorique. Mais "Ce que voit un observateur [...] dépend en partie de son expérience passée, de ses connaissances et de ses attentes " ${ }^{37}$ (p. 54): l'observateur est agi par sa propre histoire. Peu importe ce qu'il fait pour tenter de se soustraire à cette évidence, elle le rattrape dans chacun de ses jugements et ouvre, à partir des faits, aux infinis de la fabulation. Bleu, qui aspire à être seulement un oil qui voit, est déjoué par le retour constant de son passé (sous la forme de réminiscences concernant son père ou son noviciat sous le tutorat de Brun) et de son avenir (sous la forme de fantasmes dont l'objet est la "future $M^{\text {me }}$ Bleu "). Les histoires que Bleu se raconte sur la personne de Noir viennent brouiller sa perception de celui-ci, tant et si bien qu'il devient impossible de connaitre les seuls faits de son existence, qui se transforme dès lors en un écheveau inextricable de récits et d'intrigues mêlés. Bleu, lorsqu'il songe à inclure à ses rapports les histoires qu'il se raconte sur le compte de Noir, passe près de saisir ce que sa démarche a de problématique.

Lorsque Bleu, à l'image de Wakefield passant le seuil de sa maison, quitte sa chambre et part pour la Chine, nous ne saurons plus rien de lui. Il a peut-être pris conscience de l'impossibilité de réaliser la correspondance rêvée par les transcendantalistes et les inductivistes, dans un monde où le langage refuse d'adopter l'exact contour des choses, et où les fils de nos histoires composent un inextricable mélange d'où il est impossible de tirer ces ficelles qui donnent aux pantins que nous sommes l'illusion de leur autonomie. Nous pouvons donc penser ce que nous voulons du départ de Bleu pour la Chine et, pour ma part, je persiste à croire qu'il part là-bas aussi simplement que l'on ouvre la main car "Si vous regardez votre paume droite, vous voyez une carte de la Chine: les rivières coulent vers l'est, et la plupart du reste 
50

est haut et sec; les terres arables sont comme de la boue qui s'amasse dans les lignes de votre paume "38. Ultime correspondance.

38 Annie Dillard, Encounters with Chinese Writers, Middletown (Connecticut), Wesleyan University Press, 1987 (1985), p. 23 (traduction personnelle). Il serait intéressant d'examiner le rôle qu'a pu jouer la Chine comme figure de l'autre dans la littérature américaine depuis Walden jusqu'à Moon Palace. Je profite également de cette note finale pour remercier Bertrand Gervais de l'intérêt qu'il a montré à l'égard de ce texte. 